\title{
Gestión colaborativa de modelos de ciudades 3D durante su ciclo de vida basadas en servicios en la nube
}

\author{
Iñaki Prieto ${ }^{1,2}$, Jose Luis Izkara ${ }^{1}$, Rubén Béjar ${ }^{2}$ \\ ${ }^{1}$ Smart Building and Cities Area, Division de Construccion Sostenible, Tecnalia. \\ ${ }^{2}$ Grupo de Sistemas de Información Avanzados (IAAA) \\ Instituto de Investigación en Ingeniería de Aragón (I3A) \\ Universidad de Zaragoza, Mariano Esquillor s/n, 50018, Zaragoza, Spain. \\ Tel. +34-976762707, e-mail: inaki.prieto@tecnalia.com
}

\section{Resumen}

En este artículo se presenta una aproximación a la gestión de la información urbana en 3D a lo largo del ciclo de vida de la información, desde la generación hasta su utilización, pasando por la edición y mantenimiento continuo del contenido.

\section{Introducción}

Un modelo de ciudad en 3D es una representación digital de los elementos y fenómenos que ocurren en una ciudad real. La representación de la información de la ciudad está compuesta de datos espaciales georreferenciados. Resulta especialmente relevante disponer de información a diferentes escalas (edificio, entorno urbano, ciudad). Al mismo tiempo es importante que dicho modelo combine información geométrica y semántica. Y finalmente que toda la información esté incluida o referenciada en un modelo único. Estas características permitirán manejar y gestionar los datos urbanos para ser utilizados en diferentes aplicaciones como: gestión de desastres, planificación urbana, planificación de tráfico, seguridad, telecomunicaciones, navegación, turismo, etc.

Existen diferentes alternativas para la representación de modelos de datos a nivel urbano (GIS) y a nivel de edificio. Sin embargo, la unión de conceptos de ambos entornos puede conseguirse a través de CityGML, que es una de las alternativas para crear los modelos de datos.

\section{Objetivo}

El objetivo principal es investigar mecanismos de gestión colaborativa de modelos de ciudades en 3D, durante el ciclo de vida de los modelos, basados en el desarrollo de servicios web en la nube. Estos modelos representan la base para el desarrollo de soluciones de ayuda a la toma de decisiones complejas que impliquen varios agentes y diferentes ámbitos de actuación.
El flujo de trabajo para gestionar el ciclo de vida de la información de una ciudad en 3D se puede descomponer en tres grandes tareas: generación, uso y mantenimiento.

- Generación. Consiste en capturar la información tridimensional mediante distintos métodos de digitalización 3D (escáner, fotogrametría o diseño). Después, los datos son exportados al modelo de datos estándar, en nuestro caso CityGML.

- Uso. Utilizar los datos del modelo CityGML en diferentes aplicaciones tales como: toma de decisiones, visualización 3D, simulaciones, etc. [1].

- Mantenimiento. Se trata de incorporar al modelo nueva información a medida que se obtiene. Además, es necesario actualizar la información del modelo periódicamente, evitando así que el modelo se quede desfasado y desactualizado [2].

\section{Resultados}

Los resultados del trabajo de investigación presentado en este artículo se agrupan en las siguientes líneas principales:

- Metodología de generación y actualización de modelos de ciudad en 3D, La propuesta se basa en un flujo de trabajo que aborda la creación de modelos de ciudades CityGML a partir de fuentes de datos heterogéneas, con énfasis en la generación de edificios y otros elementos de la ciudad (eg vegetación, cuerpos de agua, carreteras y mobiliario urbano), la extracción automática de información semántica a partir de reglas asociadas a la información disponible y la fase de mantenimiento de dicha información que a menudo es descuidada.

- Servicios de generación y actualización de modelos de ciudad en 3D. El trabajo colaborativo y la automatización de procesos de actualización de los datos y la importancia del mantenimiento de 
modelos una vez han sido creados son cada vez más importantes. Para ello es necesario coordinar el mantenimiento de los modelos: versionado, saber quién subió qué cambios en qué momento por qué razón. Mezclar cambios de varios etc. Especialmente en escenarios de diseño colaborativo y más si están abiertos a voluntarios. Para ello se ha avanzado en la automatización de todo lo automatizable por eficiencia y para minimizar errores "humanos". Desde simples tests de humo hasta chequeos antivandalismo en escenarios abiertos a voluntarios. Se propone la aplicación del concepto, y las herramientas, de continuos delivery (CD) a este problema (normalmente usado para software, hay también alguna iniciativa de uso para documentación técnica [3]).

En esta línea se desarrollan los servicios necesarios para generar un modelo de ciudad en 3D como: configuración del modelo, generación geométrica y generación semántica. En lo relacionado a los servicios de mantenimiento se desarrollan servicios como: semantizacion masiva, inferencia, reemplazo geométrico, procesado geométrico.

- Implementación de prototipos para la validación de los servicios y modelos. Se están desarrollando prototipos de aplicaciones de demostración del uso de los modelos y servicios generados. Tres ejemplos de aplicaciones diferentes son:

- U3DCloud: Herramienta de generación de modelos de ciudades en 3D de forma colaborativa ${ }^{1}$.

- EFFESUS: Herramienta de categorización del parque edificado ${ }^{2}$.

- ADVICE: Herramienta de ayuda a la toma de decisión para la monitorización y gestión de la ciudad ${ }^{3}$.

\section{Conclusiones}

El desarrollo de modelos de datos basados en el estándar internacional CityGML permite integrar conceptos a escala ciudad y edificio dentro del mismo modelo. El trabajo presentado en este artículo presenta una solución para la gestíón

\footnotetext{
1 http://150.241.54.19:8080/u3dcloudApp/U3DCLOUD.html

${ }^{2} 3$ dcity.tecnalia.com/EffesusAppV2/faces/EffesusAppSantiago-

EffesusSantiago. $x$ html?userId=1111\&projectId=2222\&city=effesussantiago

${ }_{3}^{3}$ http://3dcity-test.tecnalia.com/AdviceApp/AdviceAppV5-

Terrain.html?project=donostiZonaGrande
}

semiautomática de los modelos de datos urbanos en 3D a lo largo del ciclo de vida de la información. La información contenida en el modelo es única y puede utilizarse para el desarrollo de diferentes aplicaciones que cubran las necesidades de diferentes agentes (gestores urbanos, técnicos y ciudadanos).

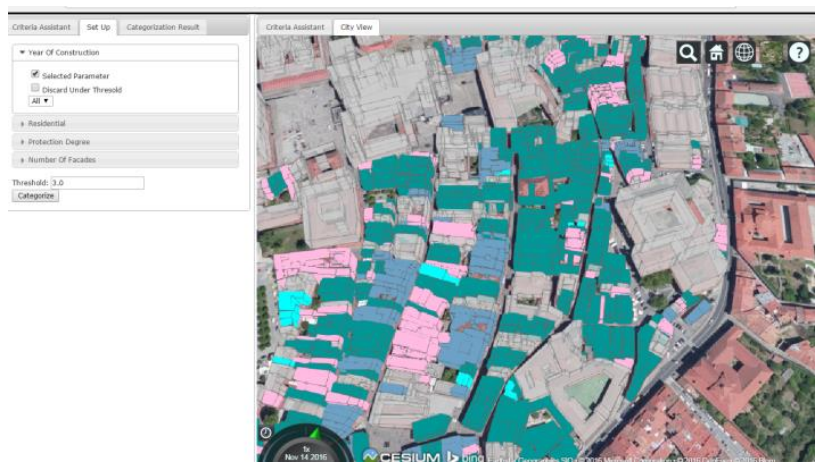

Figura 1 Herramienta de Categorizacion. Santiago de Compostela

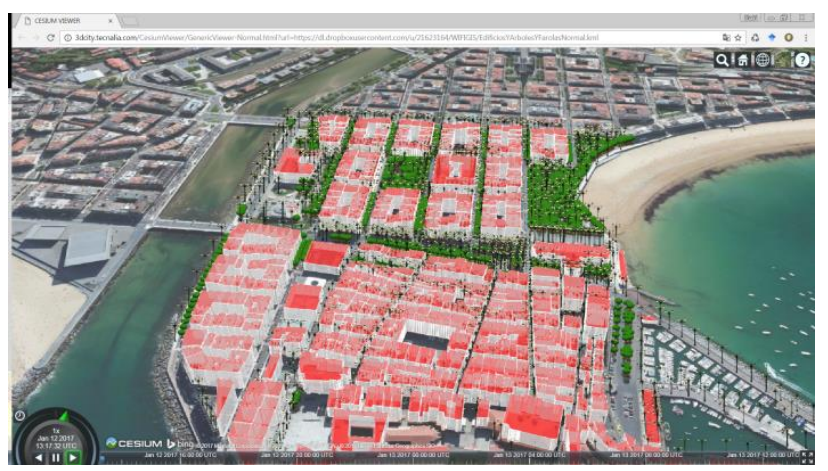

Figura 2 Herramienta ADVICE. Donostia-San Sebastián

\section{Referencias}

[1] R. Kaden and T. H. Kolbe, "City-wide total energy demand estimation of buildings using semantic 3d city models and statistical data," in ISPRS Annals. Presented at the ISPRS 8th 3DGeoInfo Conference, Istanbul, Turkey, 2013, pp. 163-171.

[2] J. Stoter, G. Vosselman, J. Goos, S. Zlatanova, E. Verbree, R. Klooster, and M. Reuvers, "Towards a National 3D Spatial Data Infrastructure: Case of The Netherlands," Photogrammetrie Fernerkundung - Geoinformation, vol. 2011. pp. 405-420, 2011.

[3] A. Gentle, "Continuous integration and delivery for documentation," 2015. [Online]. Available: https://opensource.com/business/15/7/continuous -integration-and-continuous-deliverydocumentation.

Revista “Jornada de Jóvenes Investigadores del I3A”, vol. 5 (Actas de la VI Jornada de Jóvenes Investigadores del I3A - 2 de junio de 2017). ISSN 2341-4790. 